\title{
Gustatory habituation in Drosophila relies on rutabaga (adenylate cyclase)-dependent plasticity of GABAergic inhibitory neurons
}

\author{
Pushkar Paranjpe, ${ }^{1,5}$ Veronica Rodrigues, ${ }^{1,2,4}$ K. VijayRaghavan, ${ }^{1}$ \\ and Mani Ramaswami ${ }^{1,2,3,5}$ \\ ${ }^{1}$ National Centre for Biological Sciences, Tata Institute of Fundamental Research, Bangalore 560065, India; ${ }^{2}$ Department of Biological
Sciences, Tata Institute of Fundamental Research Mumbai 400005, India; ${ }^{3}$ School of Genetics and Microbiology and School of Natural
Sciences, Smurfit Institute of Genetics and Trinity College Institute of Neuroscience, Trinity College Dublin, Dublin-2, Ireland
}

In some situations, animals seem to ignore stimuli which in other contexts elicit a robust response. This attenuation in behavior, which enables animals to ignore a familiar, unreinforced stimulus, is called habituation. Despite the ubiquity of this phenomenon, it is generally poorly understood in terms of the underlying neural circuitry. Hungry fruit flies show a proboscis extension reflex (PER) when sensory receptors are stimulated by sugars. The PER is usually followed by feeding. However, if feeding is disallowed following sugar stimulation, PER is no longer robust, and the animal is considered to be habituated to this stimulus. Our results suggest that PER habituation requires an adenylate cyclase-dependent enhancement of inhibitory output of GABAergic neurons in the subesophageal ganglion (SOG), which mediates PER. GABA synthesis in and release from glutamic acid decarboxylase (GADI) expressing neurons is necessary, and GABA $A$ receptors on cholinergic neurons are required for PER habituation. The proposed inhibitory potentiation requires glutamate/NMDAreceptor signaling, possibly playing a role in stimulus selectivity. We explain why these data provide significant and independent support for a general model in which inhibitory potentiation underlies habituation in multiple neural systems and species.

[Supplemental material is available for this article.]

An animal's environment provides a constant, dynamic multimodal sensory experience. However, from this rich input, only a subset of salient stimuli may be pertinent to the animal's survival at any time. Habituation, a phenomenon most simply defined as a reduced behavioral response to a frequently experienced, unreinforced stimulus, allows proven nonsalient stimuli to be ignored (Rankin et al. 2009; Wilson 2009). Thus, neural mechanisms of habituation, by contributing to the filtering of familiar and historically inconsequential sensory information, may allow additional cognitive resources to be focused on salient events potentially more relevant for survival.

In order to understand the molecular, cellular, and circuit mechanisms that underlie habituation, many studies have focused on sensory systems such as vision, touch, sound, smell, and taste (Pinsker et al. 1970; Duerr and Quinn 1982; Engel and Wu 1996; Quian Quiroga and van Luijtelaar 2002; Scheiner et al. 2004; Das et al. 2011). In particular, a large number of habituation studies have been reported in Drosophila (Engel and Wu 2009). While habituation research in larger model systems has offered some cellular physiological insight (Fischer and Carew 1993; Bristol and Carew 2005), studies in Drosophila, uniquely possible at a finer level due to the availability of powerful genetic tools and mutant lines, have extended our knowledge to include molecular require-

\author{
${ }^{4}$ Deceased. \\ ${ }^{5}$ Corresponding author \\ E-mail pushkarp@ncbs.res.in \\ E-mail mani.ramaswami@tcd.ie \\ Article is online at http://www.learnmem.org/cgi/doi/10.1101/lm.026641.112.
}

ments (Gailey et al. 1982; Rees and Spatz 1989; Asztalos et al. 1993, 2007; Bouhouche et al. 1993; Engel and Wu 1996, 1998; Mihalek et al. 1997; Engel et al. 2000; Megighian et al. 2001; Godenschwege et al. 2004; Zordan et al. 2005; Acevedo et al. 2007; Joiner et al. 2007; Wolf et al. 2007). Molecules identified in these studies have been shown to underlie associative learning as well as habituation, thereby unifying these two different forms of plasticity. For instance, the cAMP signaling components rutabaga and dunce are required not only for olfactory aversive conditioning but also for habituation to visual, olfactory, taste, and mechanical stimulation (Duerr and Quinn 1982; Tully and Quinn 1985; Engel and Wu 2009). However, the localized cellular requirement for the synaptic plasticity furnished by these molecules differs, depending on the type of learning undertaken by the animal.

Recent studies of Drosophila olfactory habituation have gone further to identify specific olfactory circuit elements in which many of these molecules are required (Das et al. 2011). In particular, the rutabaga adenylate cyclase is required specifically in GABAergic inhibitory local interneurons (LNs) of the antennal lobe, where the vesicular glutamate transporter, VGLUT is also required. Consistent with a model in which LNs release GABA and glutamate onto olfactory projections neurons (PNs), both $R d l$ encoded $\mathrm{GABA}_{\mathrm{A}}$ receptors and NMDARs are required in PNs for olfactory habituation but not for the basal olfactory response. Together with supporting optophysiological and imaging studies, these observations pointed to a specific mechanism for plasticity of inhibitory LNs that could underlie behavioral changes associated with olfactory habituation (Das et al. 2011). Is inhibitory 
potentiation a specific peculiarity of the olfactory circuit, or could inhibitory potentiation be a general mechanism of habituation across different sensory circuits? This is a significant question, as circuit mechanisms of habituation remain generally poorly understood. Here, we describe experimental observations in the Drosophila gustatory system which support the idea that inhibitory potentiation could be a widely used mechanism for behavioral habituation.

We analyze habituation of the appetitive taste response and robustly establish it as a tractable preparation connecting the molecular-genetic aspects of defined circuit-elements to animal behavior. Such an accessible preparation, which allows the study of plasticity from stimulus to motor-response, is of general value if it operates under conserved molecular and physiological rules. The taste response of Drosophila and other dipterans can be readily assessed by studying their feeding behaviors (Dethier 1976). The adult Drosophila has taste bristles on its leg tarsal segments (Stocker 1994; Singh 1997; Wang et al. 2004), and their contact with a tastant activates the gustatory receptor neurons housed inside these taste bristles (Marella et al. 2006). Hungry flies respond to sugar stimulation of these bristles by extension of their proboscis, the insect feeding-organ (Dethier 1976; Wang et al. 2004; Shiraiwa and Carlson 2007), and this behavior is referred to as the proboscis extension reflex (PER). PER is a robust behavior that can be readily quantified (Wang et al. 2004). However, in response to chronic tarsal stimulation, PER is attenuated (Duerr and Quinn 1982; Scheiner et al. 2004), and we show here that this decrement has properties characteristic of habituation (Rankin et al. 2009).

We confirmed that rutabaga mutants that are known to be defective for cAMP signaling (Zars et al. 2000a; Blum et al. 2009) show defects in our modified PER habituation paradigm, similar to the results of Duerr and Quinn (1982). We went further and asked whether cAMP signaling is specifically required in inhibitory or excitatory neurons using the broadly expressing genetic Gal4 drivers (Brand and Dormand 1995). We further asked whether transmitter release from GABAergic neurons and the effects of both GABA and glutamate on cholinergic neurons is necessary for gustatory habituation.

We find that rutabaga activity in GAD1-Gal4 positive neurons as well as GABA and glutamate release from these neurons is necessary for PER habituation. Moreover, both $\mathrm{GABA}_{\mathrm{A}}$ and NMDAtype glutamate receptors are necessary in Cha-Gal4 positive neurons for PER habituation. These results support a model in which enhanced inhibition onto excitatory neurons of the PER pathway mediates gustatory habituation.

\section{Results}

\section{Habituation of proboscis extension reflex}

Naive flies respond reliably to $2 \%$ sucrose stimulation on the prothoracic tarsus by exhibiting a robust proboscis extension reflex (Fig. 1B). Exposing these flies for $10 \mathrm{~min}$ to $10 \%$ sucrose
A
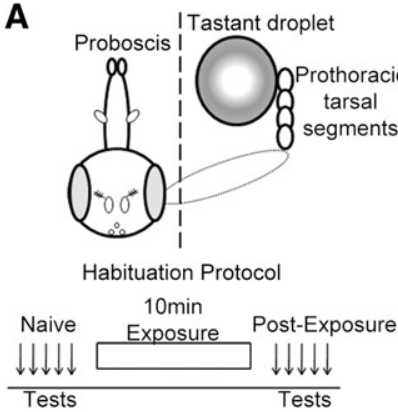

E

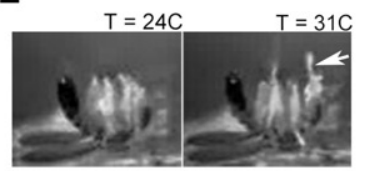

Genotype: Gr5a>TRPA1
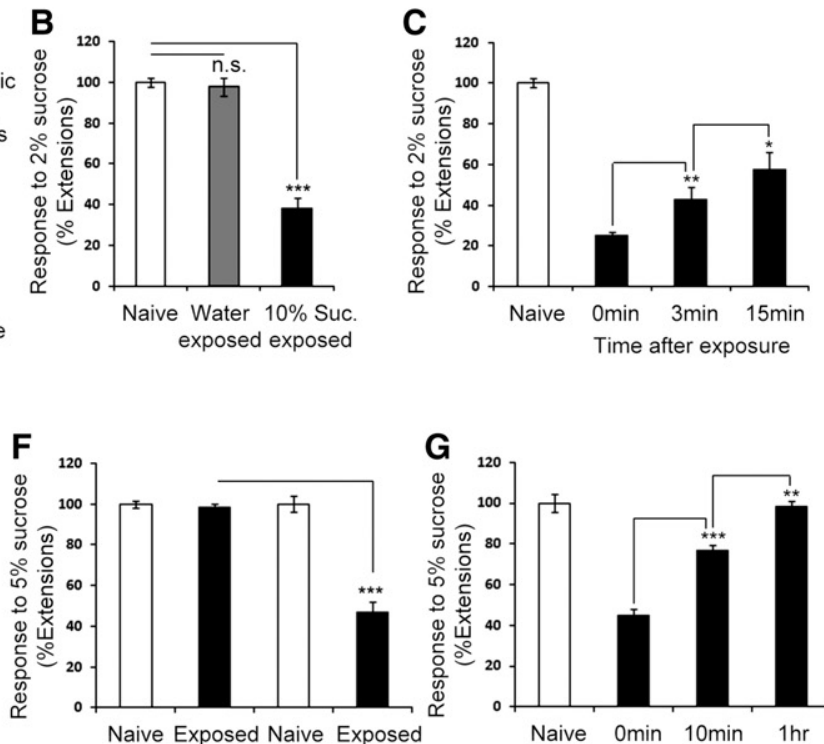

UAS-TRPA1/+ sens>TRPA1
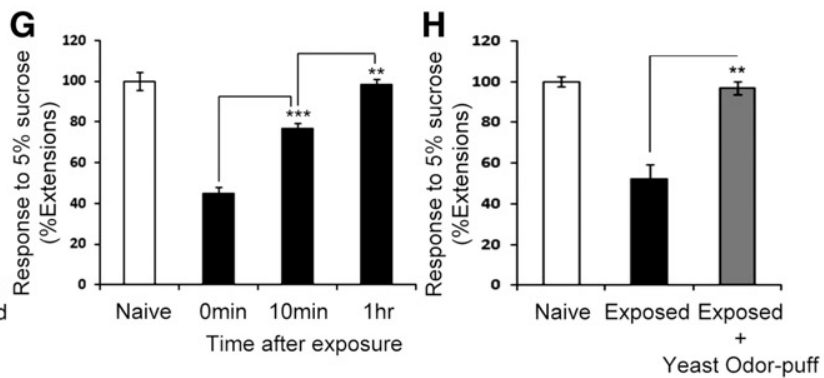

Figure 1. Habituation of proboscis extension reflex (PER). (A) Tarsal stimulation evoked PER behavior and protocol to induce and test PER habituation. Five tests, each of which is a 2 -sec touch (2\% sucrose) to the tarsal hair, are given to the naive fly. A 10-min exposure to $10 \%$ sucrose is followed by five post-exposure tests. PER in response to these naive and post-exposure tests is noted. (B) Sucrose exposure (as detailed in $A$ ) results in flies showing a significantly reduced PER (black filled bar) when compared to naive flies (open bar) ( $t$-test, $t_{(5)}=10.71, P<0.001$ ). Water-exposed flies (grey filled bar) display a nonsignificant change in post-exposure PER behavior ( $t$-test, $t_{(6)}=0.47, P=0.65$ ). (C) PER habituation recovers spontaneously, and by $15 \mathrm{~min}$, the response to $2 \%$ sucrose is about half as much as the naive response. A one-way repeated measure ANOVA shows a significant difference between naive (open bar) and post-exposure PER values (black filled bars) $(F=61.376, P<0.001)$. Post-hoc testing showed a significant difference between 0 - and 3-min recovered flies $(P<0.01$; Student-Newman-Keuls [SNK]) and 3- and 15-min recovered flies $(P<0.05 ;$ SNK). (D) Brief puffs of yeast odor just before post-exposure testing causes dishabituation (grey filled bar) ( $t$-test, $t_{(8)}=8.91, P<0.001$ ). (E) A starved Gr5a $>$ TRPA1 animal shows PER upon shifting to $31^{\circ} \mathrm{C}$ (right panel). White arrow points to the extended proboscis. $(F)$ Induction of PER habituation by TRPA1-mediated direct depolarization of sensory neurons. Open bars and black filled bars, respectively, represent PER to $5 \%$ sucrose before and after a 10-min exposure to $31^{\circ} \mathrm{C}$. (G) Habituation induced by direct depolarization recovers spontaneously. A one-way repeated measure ANOVA shows a significant difference between naive and post-exposure PER values of sens $>$ TRPA1 flies $(F=46.000, P<0.001)$. Post-hoc testing showed a significant difference between 0 and 10-min recovered flies $(P<0.001$; SNK) and 10-min and 1-hr recovered flies $(P=0.002$; SNK). $(H)$ Brief puffs of yeast odor just before post-exposure testing causes dishabituation of TRPA1-mediated habituation (grey filled bar) ( $t$-test, $\left.t_{(6)}=5.85, P<0.01\right)$. Bars represent PER mean values $( \pm S E M)$ normalized to respective naive values. $\left({ }^{*}\right) P<0.05,\left({ }^{* *}\right) P<0.01,\left({ }^{* *}\right) P<0.001,($ n.s. $)$ not significant, as determined by Student's $t$-test or SNK test. 
stimulation results in a significant response decrement when compared to untreated controls $\left(t\right.$-test, $\left.t_{(5)}=10.71, P<0.001\right)$ (Fig. 1B; raw values in Table 1). This result is similar to the gustatory habituation findings of others in the literature (Duerr and Quinn 1982; Le Bourg 1983; Fois et al. 1991; Scheiner et al. 2004). Furthermore, flies that are exposed briefly to just water display a nonsignificant change in post-exposure PER behavior ( $t$-test, $t_{(6)}=0.47, P=0.65$ ) (Fig. 1B). Thus, it is the preexposure to sugar that brings about the attenuation of the subsequently tested PER to sugar.

The attenuated PER response of sugar-exposed animals recovers spontaneously to about half the naive level in $15 \mathrm{~min}$ (Fig. 1C). A one-way repeated measure ANOVA shows a significant difference between naive and post-exposure values $(f=61.376$, $P<0.001$ ). Post-hoc testing showed a significant difference between 0 - and 3-min recovered flies $(q=4.326, P<0.01$; StudentNewman-Keuls [SNK] test) and 3 - and 15-min recovered flies $(q=3.626, P<0.05 ;$ SNK test). Spontaneous recovery suggests that the observed reduction in PER is unlikely to be due to sensory injury (if any) resulting from prolonged exposure to the tastant solution. More strikingly, exposure to brief puffs of yeast odor (see Materials and Methods) just before post-exposure testing leads to an instantaneous partial recovery of PER ( $t$-test, $t_{(8)}=$ 8.91, $P<0.001$ ) (Fig. 1D), similar to the results of Le Bourg (1983) and Fois et al. (1991). The observed "dishabituation" indicates that motor fatigue is unlikely to underlie the attenuation in PER (Thompson and Spencer 1966; Rankin et al. 2009). Consistent with prior literature, we hereafter refer to the decrement of PER observed in post-exposure testing as "PER habituation."

Olfactory response habituation can be induced without ligand-receptor interactions in the olfactory sensory neurons by using TRPA1 (Das et al. 2011), where TRPA1 is a heat-activated cation channel (Pulver et al. 2009). Direct depolarization of sweetsensitive taste receptor neurons can be induced by exposure to $31^{\circ} \mathrm{C}$ of $\mathrm{Gr} 5 a>$ TRPA1 flies (Fig. 1E). Successful activation of sweet taste neurons is evinced by the PER evoked in starved Gr5a> TRPA1 animals upon shifting them briefly to $31^{\circ} \mathrm{C}$ (Fig. 1E; Supplemental Movie M1; Materials and Methods). This approach allowed us to stimulate gustatory sensory neurons in a manner independent of the normal tastant-receptor mode of taste-neuron activation. After $10 \mathrm{~min}$ at $31^{\circ} \mathrm{C}, G r 5 a>T R P A 1$ animals show habituation to trehalose stimuli, and this decrement in behavior exhibits spontaneous recovery and dishabituation (Supplemental Fig. S2A,B). However, this treatment of Gr5a > TRPA1 flies did not lead to habituation to sucrose stimuli (data not shown), possibly due to the presence of sucrose-sensitive cells that are not labeled by Gr5a-Gal4 (Wang et al. 2004). Therefore, we used a more broadly expressing sensory neuron driver sens-Gal4 (Suster and Bate 2002) and exposed sens $>$ TRPA1 animals to $31^{\circ} \mathrm{C}$ for $10 \mathrm{~min}$ before post-exposure testing with sucrose. Indeed, this treatment resulted in a significant response decrement when compared to the UAS-TRPA1 control flies ( $t$-test, $t_{(5)}=13.26, P<$ 0.001) (Fig. 1F). Thus, sensory neuronal activation using TRPA1 is able to substitute for a real sugar stimulus as an agent that induces habituation. This mode of habituation induced by direct depolarization, too, shows the property of spontaneous recovery, and by $1 \mathrm{hr}$, the recovery is almost complete (Fig. 1F). A one-way repeated measure ANOVA shows a significant difference between naive and post-exposure PER values of sens $>$ TRPA1 flies $(f=$ $46.000, P<0.001$ ). Post-hoc testing showed a significant difference between 0 - and 10-min recovered flies $(P<0.001$; SNK) and 10 -min and 1 -hr recovered flies $(P=0.002 ; \mathrm{SNK})$. Habituation induced using TRPA1 can also be dishabituated by yeast odor exposure ( $t$-test, $t_{(6)}=5.85, P<0.01$ ) (Fig. $1 \mathrm{H}$ ), just like sugarinduced PER habituation. Additional control experiments rule out possible confounds due to unintended activation of Gr66a- marked bitter sensory neurons (Supplemental Fig. S3). Thus, PER habituation induced by forced sensory neuron depolarization is similar to that induced by sugar exposure. This indicates that the cellular site for plasticity that underlies PER habituation is downstream from sensory neuron depolarization.

\section{rutabaga is required in GAD1-Gal4 neurons for PER habituation}

Previous work has shown that rutabaga hypomorphic mutant flies show significantly reduced PER habituation (Duerr and Quinn 1982). Under our experimental conditions, while wild-type flies show robust PER habituation (Fig. 1B-D), rut ${ }^{2080}$ animals show a significantly lower amount of habituation ( $t$-test, $t_{(4)}=9.69$, $P<0.001$ ) (Supplemental Fig. S4A). Animals with the rescuing construct rut ${ }^{2080}$; UAS-rut ${ }^{+} /+$also show a statistically lower amount of habituation compared to wild-type animals ( $t$-test, $t_{(4)}=12.05, P<0.001$ ) (Supplemental Fig. S4A). Typically, across neural systems in Drosophila and for both associative as well as nonassociative learning, the cellular site of rutabaga activity holds the clue to the mechanism underlying the plasticity seen in the respective circuit (Tully and Quinn 1985; Zars et al. 2000a; Blum et al. 2009; Larkin et al. 2010; Das et al. 2011). In order to identify the cellular site in the gustatory pathway where rutabaga function may be required for PER habituation, we carried out genetic rescue experiments using a set of cell-type-restricted Gal4 lines and the $U A S-r u t^{+}$rescuing transgene. We crossed rut $^{2080}$; UAS-rut ${ }^{+}$female flies to male flies that were either control wild-type or homozygous for various Gal4 lines and assessed the male progeny for levels of PER habituation (Fig. 2A).
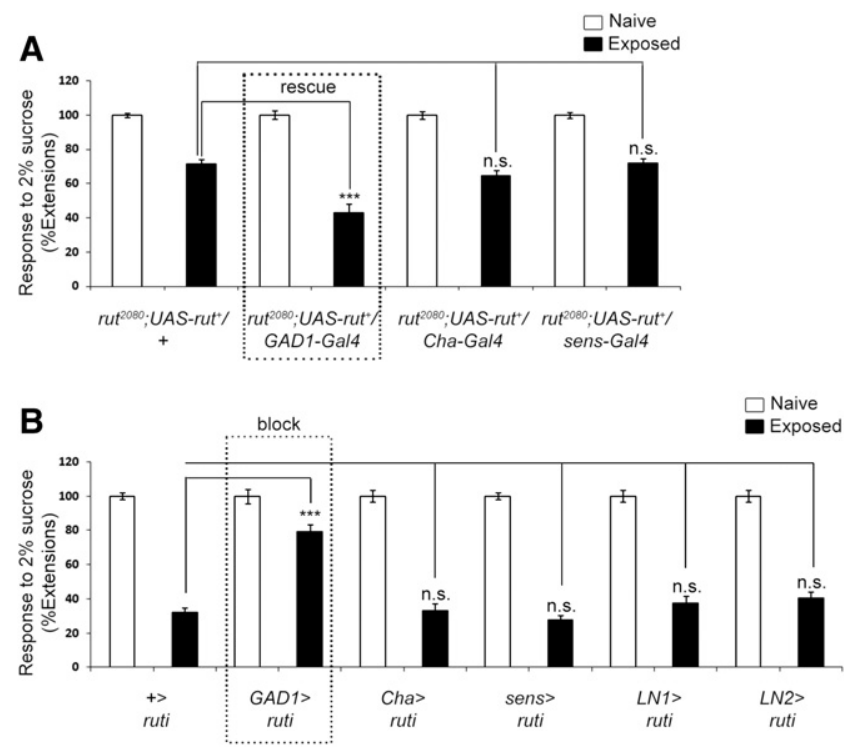

Figure 2. The rut-encoded adenylate cyclase is required in GABAergic neurons for PER habituation. GAD1 predominantly marks GABAergic neurons (Okada et al. 2009), and many of these are found in the vicinity of the subesophageal ganglion (Supplemental Fig. S5; Gordon and Scott 2009). (A) Histogram shows the efficiency of PER habituation of rut ${ }^{2080}$ flies expressing a WT rut ${ }^{+}$transgene (Zars et al. 2000b) in different classes of neurons. (B) RNAi-mediated knockdown of rut using a validated transgenic RNAi construct (VDRC101759) (Pan et al. 2009) in GAD1 neurons blocks PER habituation. Similar results obtained with an independent rut RNAi line, VDRC5569, are not shown. Additional GAL4 alone controls are shown in Supplemental Figure S4. Open bars indicate PER of naive flies; black filled bars indicate response after $10 \%$ sucrose exposure for $10 \mathrm{~min}$. Bars represent PER mean values ( \pm SEM) normalized to respective naive values. $\left.{ }^{* * *}\right) P<0.001$, (n.s.) not significant, as determined by Student's t-test. 
GAD1-Gal4 predominantly labels GABAergic neurons in the fly brain (Okada et al. 2009). Remarkably, rut ${ }^{2080}$ flies selectively expressing wild-type rutabaga in GAD1-Gal4 positive cells show a statistically improved level of PER habituation compared to control $\mathrm{rut}^{2080} / Y$; UAS-rut ${ }^{+} /+$animals $\left(t\right.$-test, $\left.t_{(6)}=5.33, P<0.01\right)$ (Fig. 2A). Cha-Gal4 predominantly labels excitatory interneurons in the fly brain (Yasuyama and Salvaterra 1999; Kitamoto 2001), and sens-Gal4 is expressed across sensory neurons (Suster and Bate 2002). However, PER habituation in rut ${ }^{2080} / Y$; UAS-rut ${ }^{+} /$ Cha-Gal4 and rut ${ }^{2080} / Y$; UAS-rut ${ }^{+} /+$; sens-Gal4/+ animals is not significantly different from rut ${ }^{2080} / Y$; UAS-rut ${ }^{+} /+$controls ( $t$-test, $t_{(5)}=2.19, P=0.06$ and $t_{(5)}=0.13, P=0.89$, respectively) (Fig. 2A). Thus, restoring wild-type rutabaga-dependent cAMP signaling in GAD1-Gal4 expressing neurons restores wild-type level PER habituation, but Cha-Gal4- and sens-Gal4-mediated rutabaga restoration shows mutant level habituation. These results support a model where rutabaga function in GAD1-Gal4 positive GABAergic inhibitory neurons, but not excitatory interneurons or sensory neurons, drives habituation of the PER pathway.

We extended these experiments by performing RNAi-mediated knockdown which was driven by the cell-type-specific Gal4s used above. We used a validated RNAi transgene against rut to knockdown endogenous rutabaga (Pan et al. 2009; Das et al. 2011) in different classes of neurons and assess its effect on habituation. PER habituation in the case of GAD1-Gal4-mediated RNAi knockdown of rutabaga is significantly reduced compared to the UAS-RNAi control stock ( $t$-test, $t_{(8)}=8.85, P<0.001$ ) (Fig. 2B). In contrast, RNAi-mediated knockdown using Cha-Gal4 and sens-Gal4 is not statistically different when compared to the $U A S$-RNAi control flies ( $t$-test, $t_{(7)}=0.55, P=0.6$ and $t_{(10)}=2.18$, $P=0.06$, respectively). All the Gal4 control animals habituate as well as wild-type animals (Supplemental Fig. S4B). These RNAibased knockdown experiments are consistent with rutabaga genetic rescue experiments and are, hence, unlikely to be due to offtarget effects of the RNAi transgene. They support our conclusion that rutabaga-dependent plasticity of GABAergic neurons is fundamental for habituation of the gustatory pathway, as previously observed in the case of olfactory habituation (Das et al. 2011). Further, for olfactory habituation, $r^{+} t^{+}$function was required exclusively in the LN1 subtype of the GABAergic local circuit interneuron of the antennal lobe (Das et al. 2011). However, PER habituation is comparable to wild type when rut is knocked down in $L N 1$ cells or in $L N 2$ cells ( $t$-test, $t_{(8)}=1.52, P=0.17$ and $t_{(8)}=2.12, P=0.07$, respectively) (Fig. 2B). Thus, rut is required in an alternative class of GAD1 expressing cells, presumably in the taste center, for PER habituation. This class is not restricted to $L N 1$ or $L N 2$ expressing neurons (Fig. 2B; Supplemental Fig. S5).

\section{NMDAR signaling is required for PER habituation}

Given that both gustatory and olfactory habituation require rutabaga function in GAD1-Gal4 neurons (Das et al. 2011), we further explored whether two other requirements for olfactory habituation, namely NMDAR function in cholinergic excitatory projection neurons and vesicular glutamate transporter (VGLUT) activity in GABAergic local interneurons (Das et al. 2011), were also true for PER habituation.

We first asked whether NMDAR function was necessary in excitatory, cholinergic interneurons. As there are no definitive Gal4-lines that mark gustatory excitatory interneurons, we used the Cha-Gal4 line, broadly expressed in all populations of excitatory interneurons (Supplemental Fig. S5; Yasuyama and Salvaterra 1999; Kitamoto 2001; Yasuyama et al. 2003), for these experiments. We crossed the NMDAR-targeting RNAi transgenes UAS-dsNR1 and UAS-dsNR2 (Xia et al. 2005; Das et al. 2011) into a Cha-Gal4 background and assessed PER habituation in these.
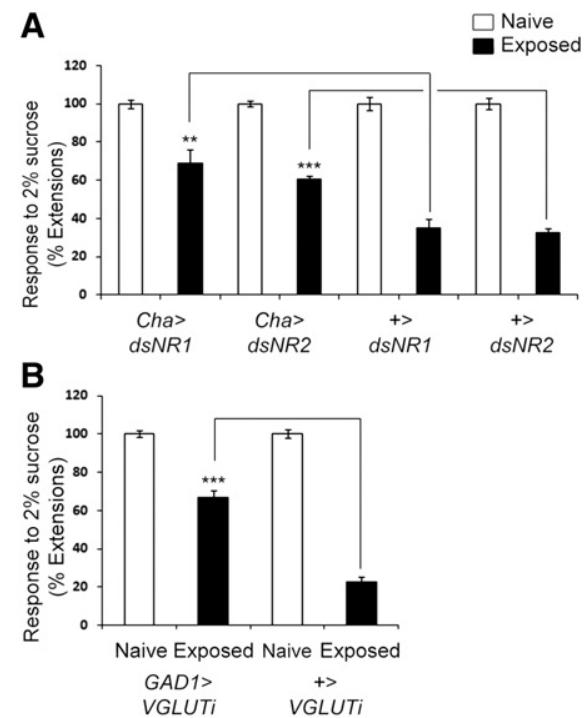

Figure 3. NMDAR signaling is required for PER habituation. (A) RNAi-mediated knockdown of NMDAR subunits NR1 and NR2 using Cha-Gal4 and its effect on PER habituation. Appropriate UAS alone control data is also included. (B) RNAi-mediated knockdown of Drosophila vesicular glutamate transporter using GAD1-Gal4 and its effect on PER habituation. Appropriate UAS alone control data is also included. Additional Gal4 alone controls are shown in Supplemental Figure S4B. Open bars indicate PER of naive flies; black filled bars indicate response after $10 \%$ sucrose exposure for $10 \mathrm{~min}$. Bars represent PER mean values $\left( \pm\right.$ SEM) normalized to respective naive values. $\left({ }^{* *}\right) P<0.01,\left({ }^{* * *}\right)$ $P<0.001$, as determined by Student's $t$-test.

As shown in Figure 3A, both Cha $>d s N R 1$ and Cha $>d s N R 2$ animals show a reduced extent of habituation which is significantly different from UAS-RNAi control flies ( $t$-test, $t_{(5)}=4.37, P<0.01$ and $t_{(8)}=9.68, P<0.001$, respectively) (Fig. $3 \mathrm{~A}$ ). This supports the idea that NMDA receptors may, indeed, have a role to play in PER habituation in cholinergic excitatory neurons, with the caveat that these neurons could theoretically be localized outside the subesophageal ganglion (SOG), a primary sensory center for gustatory behavior. Our conclusions, being based on two independent NMDAR RNAi constructs (Wu et al. 2007), is also supported by experiments that perturb glutamate release in cells that include predicted presynaptic neurons.

To further ask whether inhibitory neurons could be a source of glutamate, we expressed RNAi against Drosophila vesicular glutamate transporter (DVGLUT) RNAi (Das et al. 2011) in GAD1Gal4 positive cells and assessed the effect on PER habituation. $D V G L U T$ knockdown in GAD1 cells gives a significantly reduced PER habituation compared to UAS-RNAi control flies ( $t$-test, $t_{(7)}=10.41, P<0.001$ ) (Fig. 3B). This observation is broadly consistent with previous proposals that glutamate corelease from GABAergic neurons may be crucial for NMDAR-dependent plasticity of GABAergic synapses (Noh et al. 2010; Das et al. 2011; El Mestikawy et al. 2011; Szabadits et al. 2011). DVGLUT RNAi was obtained from VDRC and has no reported off-target effects. However, our observations do not provide any specific support for such a cellular model, given that the inhibitory and excitatory neurons relevant to the PER pathway remain so poorly defined.

\section{GABA release from GAD1-Gal4 neurons is required for PER habituation}

A model in which increased GABA release from GAD1-Gal4 cells underlies PER habituation posits that transient blockade of 
GABA release should make habituated flies behave like naive animals. We tested this prediction in Drosophila expressing a dominant, temperature-sensitive allele of dynamin (Shibire ${ }^{t s 1}$ ) in GAD1-Gal4 cells (Kitamoto 2001; Das et al. 2011). In these flies, dynamin would be expected to be active in GAD1-Gal4 neurons at room temperature but inactivated rapidly upon exposure to $32^{\circ} \mathrm{C}$, thereby reducing transmitter release from this population of neurons (Kitamoto 2001).

After exposing flies to $10 \%$ sucrose for $10 \mathrm{~min}$ at a permissive temperature $\left(23^{\circ} \mathrm{C}\right)$, we measured the PER responses of GAD1 > $S h i^{t s 1}$ animals at either the permissive or nonpermissive $\left(32^{\circ} \mathrm{C}\right)$ temperature. GAD1 $>S h i^{t s 1}$ flies show reduced PER habituation at the nonpermissive temperature compared to PER habituation seen at the permissive temperature ( $t$-test, $t_{(8)}=2.19, P<0.001$ ) (Fig. 4A). Temperature alone did not significantly affect the postexposure responses of $U A S-S h i^{t s 1}$ control flies ( $t$-test, $t_{(8)}=0.89$, $P=0.39$ ) (Fig. 4A). Similarly, the naive responses of GAD1Gal4 $>U A S-S h i^{t s 1}$ animals at permissive and nonpermissive temperatures were not significantly different from each other (data not shown). Thus, transmitter release from GAD1-Gal4 cells is es-
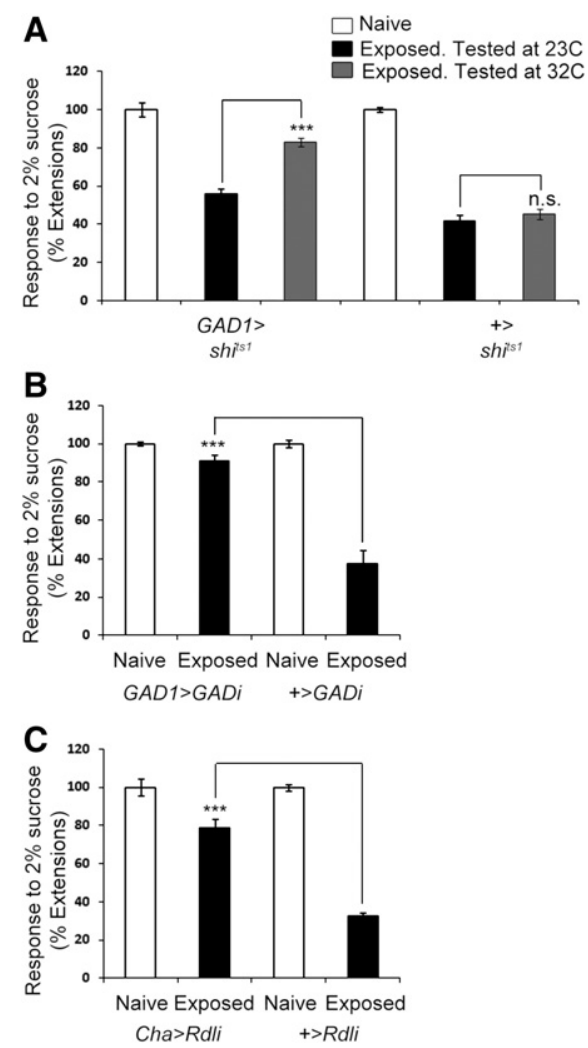

Figure 4. GABA release from GAD1-Gal4 neurons is required for PER habituation. (A) Histogram shows the efficiency of PER after $10 \%$ sucrose exposure for $10 \mathrm{~min}$ in $G A D 1>S h i i^{t s 1}$ animals tested at the permissive $\left(23^{\circ} \mathrm{C}\right.$, black filled bar) and restrictive temperatures $\left(32^{\circ} \mathrm{C}\right.$, grey filled bar) compared to PER of naive flies (open bar). Appropriate UAS alone control data is also included. (B) RNAi-mediated knockdown of GAD1 in GABAergic neurons and its effect on PER habituation. Appropriate UAS alone control data is also included. (C) RNAi-mediated knockdown of $G A B A_{A}$ receptor (Rdl) in cholinergic neurons and its effect on PER habituation. Appropriate UAS alone control data is also included. Additional Gal4 alone controls are shown in Supplemental Figure S4B. Open bars indicate PER of naive flies; black filled bars indicate response after $10 \%$ sucrose exposure for $10 \mathrm{~min}$. Bars represent PER mean values ( \pm SEM) normalized to respective naive values. $\left(^{* * *}\right) P<0.001$, (n.s.) not significant, as determined by Student's $t$-test. sential for the display of habituated behavior during the postexposure testing phase of the PER habituation paradigm.

We verified the results of the above experiment in two independent experiments detailed below. In order to provide additional evidence that GABA release from GAD1-Gal4 cells is required to manifest PER habituation, we tested the effect of RNAi-mediated knockdown of GAD1, the gene that encodes the enzyme required for GABA synthesis, in these neurons (Fig. 4B). These flies show significantly reduced PER habituation as compared to the UAS-RNAi control flies ( $t$-test, $t_{(5)}=7.76, P<$ 0.001). Thus, as observed for olfactory habituation (Das et al. 2011), GABA neurotransmitter release from GAD1-Gal4 positive neurons is necessary for PER habituation. Furthermore, knockdown of the $\mathrm{GABA}_{\mathrm{A}}$ receptor in Cha-Gal4- marked excitatory neurons via a validated RNAi line, Rdli (Liu et al. 2007, 2009), also shows a statistically reduced extent of PER habituation ( $t$-test, $t_{(4)}=10.50, P<0.001$ ) (Fig. 4C). Both GAD1RNAi and Rdli transgenes which were obtained from VDRC have no reported offtarget effects. Moreover, the results of these two experiments are mutually consistent, and hence, they support the idea that GABA-Rdl-dependent inhibition of gustatory interneurons is critical for PER habituation.

\section{Discussion}

PER is a robust, single-fly behavior, which habituates reliably upon chronic sugar stimulation. Here, we establish it as a tractable system in which the powerful neurogenetic tools available in flies can be used effectively to investigate the circuit basis of habituation. Current evidence indicates that sensory neurons, interneurons of the inhibitory subtype, and motor neurons that govern the proboscis extension reflex are tightly localized within the vicinity of the SOG (Dunipace et al. 2001; Chyb et al. 2003; Wang et al. 2004; Marella et al. 2006, 2012; Gordon and Scott 2009; Manzo et al. 2012). In other words, almost all the potentially key elements underlying habituation of PER are likely to be available for analysis in one place. This compact nature of the PER circuit makes it an extraordinarily convenient preparation for the study of multisite synaptic changes in habituation. Indeed, realtime analysis of PER habituation can be attempted by the use of optophysiological and electrophysiological methods applied to the SOG (Marella et al. 2006, 2012). We discuss the conclusions which can be unequivocally drawn from our experiments, as well as the more speculative circuit models that we propose may explain PER habituation.

\section{The mechanism for PER habituation}

We propose that the potentiation of GABAergic input drives PER habituation, as summarized in the model figure (Fig. 5). Our results suggest that rutabaga adenylate cyclase brings about the plasticity of these $G A D 1$ positive neurons (LNs), resulting in enhanced release of GABA onto excitatory elements of the PER circuit. Attenuation of PER output seems to happen in an $R d l$-dependent manner. Further, we suggest that (1) the observed requirement for NMDARs in the excitatory interneurons is for the potentiation of specific LN synapses, and (2) that glutamate required for the activation of these NMDARs will be coreleased from a subpopulation of LNs in the SOG. Although GABAergic interneurons seem to synapse over both bitter- and sweet-sensing domains of the SOG (Gordon and Scott 2009), we suggest that NMDAR activation during sugar exposure would only occur in the sweet subdomain and thereby restrict plasticity to inhibitory synapses made onto the sensory relay that carries sweet-taste information. However, the interneurons of the PER circuit are difficult to selectively target using known promoter or Gal4 driver lines. Therefore, in our 


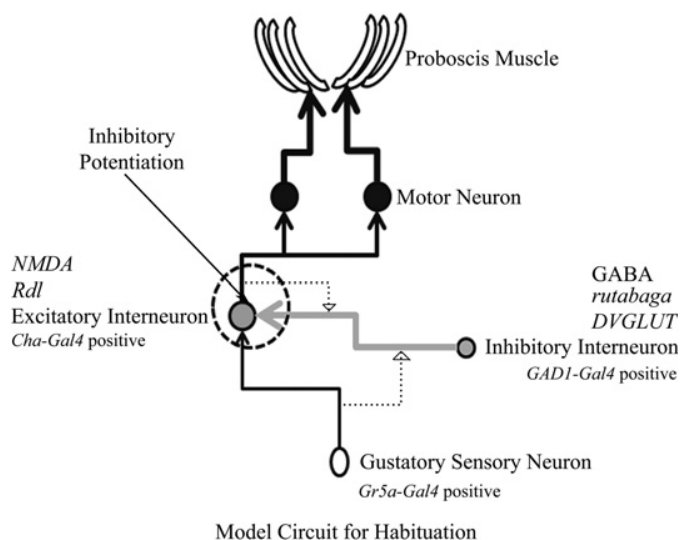

Figure 5. Model for gustatory habituation as assayed by the proboscis extension reflex paradigm. Potentiation of GABAergic synapses underlies gustatory habituation. As discussed in Gordon and Scott (2009), at least one layer of excitatory interneurons (projection neurons, PNs) must exist between the gustatory sensory neurons and the motor neurons that are required for PER. We propose that it is these neurons that undergo enhanced GABAergic inhibition during habituation in an $R d l$-dependent manner. Dampening of the taste signal this early in the PER circuit results in the observed behavioral attenuation. A GAD1-Gal4 positive subset of neurons possibly coreleases glutamate which is responsible for NMDA-based signaling in the PNs and is necessary for PER habituation. The dotted arrows indicate potential inputs to the inhibitory interneurons-feed-forward input from sensory neurons and feedback input from PNs (Sudhakaran et al. 2012). Additional synapses, such as the motor neuron output synapses, may also undergo change during PER habituation, but they need to be identified by further studies.

experiments to identify circuit elements in which cAMP (GABA or glutamate) signaling is required for PER habituation, we were limited to broadly expressed Gal4 lines that target different cell types of the PER circuit. GAD1-Gal4 is a driver for inhibitory GABAergic neurons (Ng et al. 2002; Gordon and Scott 2009; Okada et al. 2009). Similarly, Cha-Gal4 labels cholinergic excitatory neurons including the olfactory excitatory interneurons (Yasuyama and Salvaterra 1999; Kitamoto 2001; Yasuyama et al. 2003). Thus, our experiments only formally demonstrate requirements in these broad classes of neurons and do not identify specific anatomical subsets of interneurons in which rut, GAD1, VGLUT, NMDAR, and $R d l$ must function for PER habituation. We speculate that the cholinergic as well as GABAergic subpopulations involved in PER habituation may be localized at or near the SOG. Evidently, the SOG receives innervations from GABAergic interneurons (Supplemental Fig. S5; Gordon and Scott 2009), and we propose that it is within these yet to be identified LNs that rutabaga activity will be required for gustatory habituation. We hope that promoter lines that specifically identify subsets of LNs and excitatory interneurons localized to the SOG will be available in the future to test our model and to elaborate on the mechanism of PER habituation. It is certainly possible that synapses other than the inhibitory synapse highlighted in this study also change during the course of habituation, and this is an issue that should be explored by future studies.

\section{Inhibitory plasticity in behavioral habituation}

The most significant aspect of the mechanisms outlined in our study is the dependence of PER habituation on cAMP signaling in GABAergic inhibitory neurons (LNs). This, together with the need for transmitter release from these neurons for the display of habituation, argues that it is the potentiation of inhibitory transmission onto neurons that mediate PER that underlies gustatory habituation, similar to a previously proposed model for olfac- tory habituation in Drosophila (Das et al. 2011). The broad conservation of habituation mechanisms across olfactory and gustatory pathways in Drosophila suggest that similar mechanisms may be more widely used in biology. The key characteristic of such a mechanism is that it allows selective potentiation of inhibitory synapses made onto principle excitatory neurons (PNs). Such a component could be provided by postulating (1) a requirement for NMDA receptor activation which would only occur in active PNs, (2) the requirement for a local retrograde signal that would only be sensed by LN terminals in the vicinity of these active PNs, and (3) specific thresholds for signaling strength required to trigger the underlying process of synaptic plasticity. In the absence of neurophysiological data, the current study falls short of establishing this model for habituation in the Drosophila gustatory system, let alone in nervous systems at large.

However, there are some hints of support for such a model from other systems in which habituation has been studied using neurophysiological approaches. In Aplysia, depression of a sensory-motor synapse does not appear to be a general mechanism for habituation (Stopfer and Carew 1996). Siphon withdrawal reflex habituation induced by near threshold tail sensory neuron stimulation (T-SWR) is evident as decrementing behavioral response to tail stimulation but is not accompanied by sensorymotor depression: instead, significant facilitation of sensory neuron output is observed (Stopfer and Carew 1996). One proposal to explain this anomaly is the potentiation of inhibitory input onto interneurons that feed onto siphon motor neurons (Stopfer and Carew 1996). Furthermore, a recurrent inhibitory microcircuit which potentiates in an activity-dependent manner has been shown to exist in the T-SWR pathway and the siphon stimulatedsiphon withdrawal reflex pathway (S-SWR) (Fischer and Carew 1993). Interestingly, the habituation of T-SWR is known to get disrupted when the inhibition is disrupted pharmacologically (Bristol and Carew 2005). Similarly, the cellular basis for habituation of the crayfish escape reflex has been extensively investigated, and the most recent studies show that inhibition plays a dominant role in establishing habituation in this system (Krasne and Teshiba 1995; Shirinyan et al. 2006). The Bruce Effect (also known as the Strange Male effect) in which female rodents distinguish between the familiar male and a strange male appears to arise due a selective strengthening of inhibitory feedback onto mitral cells in the vomeronasal organ that are triggered by the scent of the familiar male (Wilson et al. 1985; Brennan et al. 1990; Gao and Strowbridge 2009).

Together, the above observations argue that it could be of value to consider that potentiation of feedback inhibition (Sudhakaran et al. 2012) has a broader role in behavioral habituation. Remarkably, the first mention of such a model appears to have been made in 1943 psychological theory, which proposed behavioral habituation may arise from a "buildup of reactive inhibition" (Hull 1943). Although the physical basis for this reactive inhibition was not considered in the original theory, it appears conceivable that feedback inhibitory circuits, now known to be ubiquitous in all brain regions, are well-positioned to mediate the process conceived by Hull. While the history of habituation as well as the arguments against Hull's construct have been considered by others (Thompson and Spencer 1966; Rankin et al. 2009), we suggest that there may be reasons to reconsider and reinterpret Hull's theory in the context of contemporary neuroscience.

\section{Materials and Methods}

\section{Drosophila stocks}

For all the experiments, Canton $S$ has been used as wild type (WT). The Drosophila stocks used were generously provided by the 
following sources: the sensory neuron Gal4 lines Gr5a-Gal4 and Gr66a-Gal4 (Wang et al. 2004) were provided by Kristin Scott, and sens-Gal4 (Suster and Bate 2002) was provided by Michael Bate; GAD1-Gal4 was from Gero Miesenbock (Oxford University, Oxford, UK) (Ng et al. 2002). The RNAi lines were provided by the following sources: $U A S-R d l R N A i^{8-10 G}$ (Rdli) lines were obtained from Ron Davis (Baylor College of Medicine, Houston, TX) (Liu et al. 2007, 2009); UAS-dsNR1, UAS-dsNR2 was from Tim Tully (Cold Spring Harbor Laboratory, Cold Spring Harbor, NY) (Xia et al. 2005; Wu et al. 2007); Cha-Gal4 and UAS-shits1 were from

Table 1. Gustatory response indices for genotypes tested to PER habituation paradigm

\begin{tabular}{|c|c|c|c|c|}
\hline Genotype & Tastant exposed & Naive & Post-exposure & $P$-value \\
\hline \multicolumn{5}{|l|}{ Tastant tested $2 \%$ sucrose (no. of flies) } \\
\hline WT $(+)$ & $10 \%$ sucrose & $93.6 \pm 2.3(25)$ & $35.5 \pm 5.6(42)$ & $5.00 \times 10^{-06}$ \\
\hline WT (+) & Water & & $91.6 \pm 4.3(48)$ & 0.65 \\
\hline \multicolumn{5}{|l|}{ Temporal recovery } \\
\hline \multirow[t]{3}{*}{ WT $(+)$} & $10 \%$ sucrose & $94.9 \pm 2.2(20)$ & 0 min: $23.8 \pm 1.9(17)$ & $4.00 \times 10^{-06}$ \\
\hline & & & 3 min: $40.3 \pm 6.3(17)$ & 0.007 \\
\hline & & & $15 \min : 54.6 \pm 8.5(17)$ & \\
\hline \multicolumn{5}{|l|}{ Dishabituation by yeast exposure } \\
\hline WT $(+)$ & $10 \%$ sucrose & $92.7 \pm 2.1(30)$ & $26 \pm 3.7(30)$ & $5.78 \times 10^{-07}$ \\
\hline & & & Post-yeast exposure & \\
\hline Tastant tested $5 \%$ sucrose (no. of flies) & Temp. exposed & & $30.9 \pm 2.3(30)$ & $4.50 \times 10$ \\
\hline UAS-TRPA1/+; sens-Gal4 & $30^{\circ} \mathrm{C}$ for $10 \mathrm{~min}$ & $88.8 \pm 3.8(25)$ & $41.8 \pm 4.8(25)$ & $1.30 \times 10^{-05}$ \\
\hline \multicolumn{5}{|l|}{ Temporal recovery } \\
\hline \multirow[t]{3}{*}{ UAS-TRPA1/+; sens-Gal4 } & $30^{\circ} \mathrm{C}$ for $10 \mathrm{~min}$ & $88.8 \pm 4.6(25)$ & $0 \min : 40 \pm 2.8(25)$ & $2.80 \times 10^{-05}$ \\
\hline & & & $10 \mathrm{~min}: 68 \pm 2.8(25)$ & $<0.001$ \\
\hline & & & $1 \mathrm{~h}: 87.2 \pm 3(25)$ & 0.002 \\
\hline UAS-TRPA1 / +; Gr66a-Gal4 & $30^{\circ} \mathrm{C}$ for $10 \mathrm{~min}$ & $80.0 \pm 7.07(25)$ & $80.0 \pm 6.28(25)$ & $1.80 \times 10^{-01}$ \\
\hline \multicolumn{5}{|l|}{ Dishabituation by yeast exposure } \\
\hline \multirow[t]{2}{*}{ UAS-TRPA1/+; sens-Gal4 } & $30^{\circ} \mathrm{C}$ for $10 \mathrm{~min}$ & $93.6 \pm 2.3(25)$ & $49 \pm 7.2(25)$ & $8.10 \times 10^{-05}$ \\
\hline & & & Post-yeast exposure & \\
\hline Tastant tested $30 \%$ trehalose (no. of flies) & Temp. exposed & & $90.6 \pm 3.3(25)$ & 0.0003 \\
\hline UAS-TRPA1/+;Gr5a-Gal4 & $30^{\circ} \mathrm{C}$ for $10 \mathrm{~min}$ & $929+483(25)$ & & \\
\hline \multicolumn{5}{|l|}{ Temporal recovery } \\
\hline \multirow{3}{*}{ UAS-TRPA1/+;Gr5a-Gal4 } & $30^{\circ} \mathrm{C}$ for $10 \mathrm{~min}$ & $92.9 \pm 4.83(25)$ & 0 min: $60.66 \pm 4.7(25)$ & $<0.001$ \\
\hline & & & $10 \min : 74.4 \pm 5.57(25)$ & 0.005 \\
\hline & & & 1 h: $90.0 \pm 1.3(25)$ & 0.002 \\
\hline \multicolumn{5}{|l|}{ Dishabituation by yeast exposure } \\
\hline \multirow[t]{2}{*}{ UAS-TRPA1/+; Gr5a-Gal4 } & $30^{\circ} \mathrm{C}$ for $10 \mathrm{~min}$ & $86.9 \pm 5.7(25)$ & $61.86 \pm 3.57(25)$ & 0.005 \\
\hline & & & Post-yeast exposure & \\
\hline Tastant tested $1 \%$ sucrose (no. of flies) & Tastant exposed & & $88.93 \pm 4.67(25)$ & $4.00 \times 10^{-03}$ \\
\hline WT $(+)$ & $\begin{array}{l}\text { lastant exposed } \\
10 \% \text { sucrose }\end{array}$ & & & \\
\hline rut2080/Y & $10 \%$ sucrose & $83.6 \pm 2.21$ (30) & $23.6 \pm 2.12(50)$ & $\begin{array}{l}5.86 \times 10^{-14} \\
268 \times 10^{-09}\end{array}$ \\
\hline rut2080/Y; UAS-rut + & & $84.0 \pm 5.09(25)$ & $69.6 \pm 3.03(25)$ & $2.68 \times 10^{-09}$ \\
\hline Tastant tested $2 \%$ sucrose (no. of flies) & $\begin{array}{l}10 \% \text { sucrose } \\
\text { Tastant exposed }\end{array}$ & $85.6 \pm 3.34(25)$ & $44.0 \pm 5.47(25)$ & $4.17 \times 10^{-03}$ \\
\hline rut $2080 / Y$ & $\begin{array}{l}\text { Tastant exposed } \\
10 \% \text { sucrose }\end{array}$ & & & \\
\hline rut2080/Y; UAS-rut + & $\begin{array}{l}10 \% \text { sucrose } \\
10 \% \text { sucrose }\end{array}$ & $92.8 \pm 2(25)$ & $75.2 \pm 4(23)$ & $\begin{array}{c}0.003 \\
1.20 \times 10^{-10}\end{array}$ \\
\hline GAD1-Gal4 > rut2080/Y; UAS-rut + & $\begin{array}{l}10 \% \text { sucrose } \\
10 \% \text { sucrose }\end{array}$ & $93.2 \pm 1.4(72)$ & $66.7 \pm 2.4(72)$ & $1.20 \times 10^{-10}$ \\
\hline Cha-Gal4 > rut2080/Y; UAS-rut + & $\begin{array}{l}10 \% \text { sucrose } \\
10 \% \text { sucrose }\end{array}$ & $87.6 \pm 2.4(23)$ & $37.8 \pm 4.9(23)$ & $1.70 \times 10^{-05}$ \\
\hline sens-Gal4 > rut2080/Y; UAS-rut + & $\begin{array}{l}10 \% \text { sucrose } \\
10 \% \text { sucrose }\end{array}$ & $90.4 \pm 2.3(25)$ & $58.4 \pm 3.4(25)$ & $2.10 \times 10^{-05}$ \\
\hline GAD1-Gal4/+ & & $93.6 \pm 1.8(25)$ & $67.2 \pm 2.8(25)$ & $2.70 \times 10^{-05}$ \\
\hline Cha-Gal4/ + & $\begin{array}{l}10 \% \text { sucrose } \\
10 \% \text { sucrose }\end{array}$ & $94.4 \pm 1.78(25)$ & $36.8 \pm 5.17(25)$ & $2.5 \times 10^{-06}$ \\
\hline & $10 \%$ sucrose & $94.4 \pm 3.34(25)$ & $30.4 \pm 4.14(25)$ & $9.07 \times 10^{-07}$ \\
\hline $\begin{array}{l}\text { sens-Gal4/+ } \\
+/ \text { rutRNAi }\end{array}$ & $10 \%$ sucrose & $92.8 \pm 2.96(25)$ & $27.2 \pm 6.54(25)$ & $7.27 \times 10^{-06}$ \\
\hline + /rutRNAi & $10 \%$ sucrose & $86.9 \pm 2(70)$ & $28 \pm 2.4(70)$ & $5.60 \times 10^{-17}$ \\
\hline GAD1-Gal4 > rutRNAi & $10 \%$ sucrose & $81.6 \pm 4.1(25)$ & $64.8 \pm 4.8(25)$ & $1.00 \times 10^{-02}$ \\
\hline Cha-Gal4 > rutRNAi & $10 \%$ sucrose & $92.8 \pm 3.3(24)$ & $30.6 \pm 4.1(24)$ & $5.90 \times 10^{-07}$ \\
\hline sens-Gal4 > rutRNAi & $10 \%$ sucrose & $91 \pm 2(23)$ & $25 \pm 3.2(23)$ & $2.60 \times 10^{-08}$ \\
\hline LN1-Gal4 > rutRNAi & $10 \%$ sucrose & $91.2 \pm 3.6(25)$ & $34.4 \pm 3.9(25)$ & $2.10 \times 10^{-06}$ \\
\hline LN2-Gal4 > rutRNAi & $10 \%$ sucrose & $91.2 \pm 3.6(25)$ & $36.8 \pm 3.8(25)$ & $2.80 \times 10^{-06}$ \\
\hline Cha-Gal4 > dsNR1 & $10 \%$ sucrose & $93 \pm 2.2(20)$ & $64 \pm 7.3(20)$ & $5.00 \times 10^{-03}$ \\
\hline Cha-Gal4 > dsNR2 & $10 \%$ sucrose & $92.8 \pm 1.7(25)$ & $56 \pm 2(25)$ & $2.60 \times 10^{-07}$ \\
\hline$+/ d s N R 1$ & $10 \%$ sucrose & $89 \pm 3.5(20)$ & $31 \pm 4.8(20)$ & $2.80 \times 10^{-05}$ \\
\hline$+/ d s N R 2$ & $10 \%$ sucrose & $92 \pm 3.2(25)$ & $30 \pm 2.2(25)$ & $9.70 \times 10^{-08}$ \\
\hline GAD1-Gal4 > DVGLUT RNAi & $10 \%$ sucrose & $95 \pm 1.7(24)$ & $63.4 \pm 3.9(24)$ & $3.00 \times 10^{-05}$ \\
\hline$+/ D V G L U T R N A i$ & $10 \%$ sucrose & $89.4 \pm 2.2(24)$ & $20.2 \pm 2.6(24)$ & $3.00 \times 10^{-08}$ \\
\hline GAD1-Gal4 > UAS-shits1 (RT) & $10 \%$ sucrose & $70.5 \pm 3.8(29)$ & $39.4 \pm 2.5(27)$ & $5.90 \times 10^{-05}$ \\
\hline GAD1-Gal4 > UAS-shits1 (32C) & $10 \%$ sucrose & $74.5 \pm 6.5(27)$ & $58.4 \pm 2.3(27)$ & $1.50 \times 10^{-02}$ \\
\hline+ /UAS-shits1 (RT) & $10 \%$ sucrose & $90.4 \pm 1.1(25)$ & $37.6 \pm 3.0(25)$ & $8.20 \times 10^{-08}$ \\
\hline+ /UAS-shits1 (32C) & $10 \%$ sucrose & $92.5 \pm 2.2(25)$ & $40.8 \pm 2.6(25)$ & $4.80 \times 10^{-08}$ \\
\hline GAD1-Gal4 > GAD1 RNAi & $10 \%$ sucrose & $97.6 \pm 1.1(25)$ & $88.8 \pm 3.0(25)$ & $1.50 \times 10^{-02}$ \\
\hline$+/ G A D 1 R N A i$ & $10 \%$ sucrose & $94.4 \pm 1.8(25)$ & $35.2 \pm 7.1(25)$ & $1.80 \times 10^{-05}$ \\
\hline Cha-Gal4 > Rdl RNAi & $10 \%$ sucrose & $94 \pm 4.4(20)$ & $74 \pm 4.4(20)$ & 0.01 \\
\hline$+/ R d l R N A i$ & $10 \%$ sucrose & $93.3 \pm 1.7(45)$ & $30.2 \pm 1.7(45)$ & $8.00 \times 10^{-15}$ \\
\hline
\end{tabular}


Toshi Kitamoto (University of Iowa, Iowa City, IA); UAS-VGLUT RNAi stock CG9887(KK) and the UAS-RutRNAi (Ruti) lines (VDRC5569 and VDRC101759) (Pan et al. 2009) were from the Vienna Drosophila Research Center. The rutabaga allele rut ${ }^{2080}$ and the UAS-rut ${ }^{+}$transgenes were obtained from Martin Heisenberg (University of Würzburg, Würzburg, Germany) (Zars et al. 2000b). UAS-TRPA1 strains were generated by Paul Garrity (Brandeis University, Waltham/Boston, MA) (Rosenzweig et al. 2005; Pulver et al. 2009), and balancer and other strains were obtained from the Bloomington Stock Center.

\section{Proboscis extension reflex behavior}

Zero- to 12-h flies were transferred to a fresh food bottle for $1 \mathrm{~d}$. Two-day-old adult, 24-h starved, Drosophila males were used throughout the study. Each animal was mounted individually on a glass slide $4 \mathrm{~h}$ before assaying and kept in a humidified chamber to prevent desiccation. Immobilization of animals was carried out dorsal side down so that PER could be observed clearly under a stereomicroscope (Fig. 1A). Except for one prothoracic leg, all other legs were glued to the glass slide. This prothoracic leg was tethered in place to enable easy and carefully timed tarsal stimulation (Fig. 1A). Stimulation consisted of a tastant solution applied using a 1-mL syringe needle to the tarsal hair. Animals were always water-satiated prior to tastant stimulation. Each stimulus lasted $2 \mathrm{sec}$. A pretest (test of the naive PER response performed "preexposure") used five consecutive tarsal stimuli. A 10-sec gap was given between successive stimuli. Weak or completely failed PER events in response to a stimulus were scored as a zero. A successful PER response to a tarsal stimulus was scored as one. Thus, for example, a successful PER of 4 of 5 stimuli in a test would result in a performance score of 0.8 .

\section{Habituation of PER}

Using the same stimulation method as above, the tarsal hair was stimulated continuously for a period of $10 \mathrm{~min}$. The leg was washed and gently dried with an absorbent piece of filter paper. This was followed by a "post-test" (response test post-exposure), which was identical in procedure to the pretest.

Dishabituation experiments made use of a plastic Pasteur pipette for blowing fermented yeast odor at the mounted animals. Three consecutive puffs were delivered at the antennae with a 2 -sec gap in between.

\section{TRPA1/heat-mediated gustatory stimulation of flies}

A water-filled glass bulb served as a heat source. Water circulating through the bulb was maintained at a constant temperature $\left(32^{\circ} \mathrm{C}\right)$ using an isothermal water bath and pump assembly. Heat stimulation was carried out by keeping the immobilized flies in contact with the warmed glass surface during pre- and posttests, or in contact for a continuous period of $10 \mathrm{~min}$ when using direct neuronal depolarization for inducing habituation. A roomtemperature surface $\left(23^{\circ} \mathrm{C}\right)$ was used to rest the flies in between successive heat stimuli.

For Gr66a > TRPA1 fly feeding experiments, a red dye $(0.1 \%)$ (Carmoisine Red; Anand Dyes and Co. Ltd.) was added to a $2 \%$ sucrose solution so that, upon feeding, flies feeding well could be readily distinguished from poorly feeding flies. A tissue paper soaked in this mixture was placed inside a falcon tube. Twentyfour-h starved flies were introduced into this falcon tube and allowed to feed for $10 \mathrm{~min}$, after which they were immobilized on ice for counting good vs. poor feeders. Flies were either allowed to feed at $32^{\circ} \mathrm{C}$ using the heating apparatus described above or at room temperature $\left(23^{\circ} \mathrm{C}\right)$. Flies used in a feeding experiment are classified into two groups-good feeders and poor feeders, and the percent fractions of these categories are reported as the result of the feeding assay.

\section{Brain dissection, immunohistochemistry, and imaging} Brains were dissected in PBS and fixed in 4\% paraformaldehyde diluted in PBS containing 0.3\% Triton-X100 (PTX) for $30 \mathrm{~min}$ at room temperature. Fixed samples were washed three times for 20 min each in PTX and then incubated in primary antibody diluted in $0.3 \%$ PTX for $48 \mathrm{~h}$ at $4^{\circ} \mathrm{C}$ on a shaker. The primary antibodies used were rabbit anti-GFP (1:10,000; Molecular Probes) and mouse anti-Bruchpilot (mAbnc82, 1:20; DSHB). After incubation, brains were washed repeatedly in $0.3 \%$ PTX and incubated in Alexa-488-, Alexa-568-, or Alexa-647-coupled secondary antibody (Molecular Probes) diluted at 1:400 in PTX. Samples were washed in PTX, mounted in $70 \%$ glycerol on a spacer slide, and imaged with Olympus Fluoview (FV1000).

\section{Acknowledgments}

We thank S. Das, M. Sadanandappa, I. Sudhakaran, C. Tabone, J. Lee, I. Twick, Dr. S. Sane, and Dr. K.S. Krishnan for advice and help throughout the course of these experiments. We also thank Kristin Scott, Michael Bate, Gero Meisenbock, Ron Davis, Tim Tully, Toshi Kitamoto, Martin Heisenberg, Paul Garrity, Gajendra, and the Bloomington and Vienna Stock Centers for fly stocks and reagents. We thank C. Tabone, S. Das, and A. Saxena for useful discussions and/or comments on the manuscript. This work was funded by grants from Science Foundation Ireland (M.R.), the Indian Department of Biotechnology (V.R.), and core funding to the Tata Institute of Fundamental Research from the Department of Atomic Energy.

\section{References}

Acevedo SF, Froudarakis EI, Kanellopoulos A, Skoulakis EM. 2007. Protection from premature habituation requires functional mushroom bodies in Drosophila. Learn Mem 14: 376-384.

Asztalos Z, von Wegerer J, Wustmann G, Dombradi V, Gausz J, Spatz HC, Friedrich P. 1993. Protein phosphatase 1-deficient mutant Drosophila is affected in habituation and associative learning. J Neurosci 13: 924-930.

Asztalos Z, Arora N, Tully T. 2007. Olfactory jump reflex habituation in Drosophila and effects of classical conditioning mutations. J Neurogenet 21: $1-18$.

Blum AL, Li W, Cressy M, Dubnau J. 2009. Short- and long-term memory in Drosophila require cAMP signaling in distinct neuron types. Curr Biol 19: $1341-1350$

Bouhouche A, Vaysse G, Corbiere M. 1993. Immunocytochemical and learning studies of a Drosophila melanogaster neurological mutant, no-bridgeKS49 as an approach to the possible role of the central complex. J Neurogenet 9: 105-121.

Brand AH, Dormand EL. 1995. The GAL4 system as a tool for unravelling the mysteries of the Drosophila nervous system. Curr Opin Neurobiol 5: $572-578$.

Brennan P, Kaba H, Keverne EB. 1990. Olfactory recognition: A simple memory system. Science 250: $1223-1226$.

Bristol AS, Carew TJ. 2005. Differential role of inhibition in habituation of two independent afferent pathways to a common motor output. Learn Mem 12: 52-60.

Chyb S, Dahanukar A, Wickens A, Carlson JR. 2003. Drosophila Gr5a encodes a taste receptor tuned to trehalose. Proc Natl Acad Sci 100: 14526-14530.

Das S, Sadanandappa MK, Dervan A, Larkin A, Lee JA, Sudhakaran IP, Priya R, Heidari R, Holohan EE, Pimentel A, et al. 2011. Plasticity of local GABAergic interneurons drives olfactory habituation. Proc Natl Acad Sci 108: E646-E654.

Dethier VG. 1976. The hungry fly. Harvard University Press, Cambridge, MA.

Duerr JS, Quinn WG. 1982. Three Drosophila mutations that block associative learning also affect habituation and sensitization. Proc Natl Acad Sci 79: 3646-3650.

Dunipace L, Meister S, McNealy C, Amrein H. 2001. Spatially restricted expression of candidate taste receptors in the Drosophila gustatory system. Curr Biol 11: 822-835.

El Mestikawy S, Wallen-Mackenzie A, Fortin GM, Descarries L, Trudeau LE. 2011. From glutamate co-release to vesicular synergy: Vesicular glutamate transporters. Nat Rev Neurosci 12: 204-216.

Engel JE, Wu CF. 1996. Altered habituation of an identified escape circuit in Drosophila memory mutants. J Neurosci 16: 3486-3499.

Engel JE, Wu CF. 1998. Genetic dissection of functional contributions of specific potassium channel subunits in habituation of an escape circuit in Drosophila. J Neurosci 18: 2254-2267.

Engel JE, Wu CF. 2009. Neurogenetic approaches to habituation and dishabituation in Drosophila. Neurobiol Learn Mem 92: 166-175. 
Engel JE, Xie XJ, Sokolowski MB, Wu CF. 2000. A cGMP-dependent protein kinase gene, foraging, modifies habituation-like response decrement of the giant fiber escape circuit in Drosophila. Learn Mem 7: 341-352.

Fischer TM, Carew TJ. 1993. Activity-dependent potentiation of recurrent inhibition: A mechanism for dynamic gain control in the siphon withdrawal reflex of Aplysia. J Neurosci 13: 1302-1314.

Fois C, Medioni J, Le Bourg E. 1991. Habituation of the proboscis extension response as a function of age in Drosophila melanogaster. Gerontology 37: 187-192.

Gailey DA, Jackson FR, Siegel RW. 1982. Male courtship in Drosophila: The conditioned response to immature males and its genetic control. Genetics 102: 771-782.

Gao Y, Strowbridge BW. 2009. Long-term plasticity of excitatory inputs to granule cells in the rat olfactory bulb. Nat Neurosci 12: 731-733.

Godenschwege TA, Reisch D, Diegelmann S, Eberle K, Funk N, Heisenberg M, Hoppe V, Hoppe J, Klagges BR, Martin JR, et al. 2004. Flies lacking all synapsins are unexpectedly healthy but are impaired in complex behaviour. Eur J Neurosci 20: 611-622.

Gordon MD, Scott K. 2009. Motor control in a Drosophila taste circuit. Neuron 61: 373-384.

Hull CL. 1943. Principles of behavior: An introduction to behavior theory. Appleton-Century, Oxford, UK

Joiner MA, Asztalos Z, Jones CJ, Tully T, Wu CF. 2007. Effects of mutant Drosophila K+ channel subunits on habituation of the olfactory jump response. J Neurogenet 21: $45-58$.

Kitamoto T. 2001. Conditional modification of behavior in Drosophila by targeted expression of a temperature-sensitive shibire allele in defined neurons. J Neurobiol 47: 81-92.

Krasne FB, Teshiba TM. 1995. Habituation of an invertebrate escape reflex due to modulation by higher centers rather than local events. Proc Natl Acad Sci 92: 3362-3366.

Larkin A, Karak S, Priya R, Das A, Ayyub C, Ito K, Rodrigues V, Ramaswami M. 2010. Central synaptic mechanisms underlie short-term olfactory habituation in Drosophila larvae. Learn Mem 17: 645-653.

Le Bourg E. 1983. Aging and habituation of the tarsal response in Drosophila melanogaster. Gerontology 29: 388-393.

Liu X, Krause WC, Davis RL. 2007. GABAA receptor RDL inhibits Drosophila olfactory associative learning. Neuron 56: 1090-1102.

Liu X, Buchanan ME, Han KA, Davis RL. 2009. The GABAA receptor RDL suppresses the conditioned stimulus pathway for olfactory learning. I Neurosci 29: 1573-1579.

Manzo A, Silies M, Gohl DM, Scott K. 2012. Motor neurons controlling fluid ingestion in Drosophila. Proc Natl Acad Sci 109: 6307-6312.

Marella S, Fischler W, Kong P, Asgarian S, Rueckert E, Scott K. 2006. Imaging taste responses in the fly brain reveals a functional map of taste category and behavior. Neuron 49: 285-295.

Marella S, Mann K, Scott K. 2012. Dopaminergic modulation of sucrose acceptance behavior in Drosophila. Neuron 73: 941-950.

Megighian A, Zordan M, Costa R. 2001. Giant neuron pathway neurophysiological activity in per(0) mutants of Drosophila melanogaster. J Neurogenet 15: 221-231.

Mihalek RM, Jones CJ, Tully T. 1997. The Drosophila mutation turnip has pleiotropic behavioral effects and does not specifically affect learning. Learn Mem 3: 425-444.

Ng M, Roorda RD, Lima SQ, Zemelman BV, Morcillo P, Miesenbock G. 2002. Transmission of olfactory information between three populations of neurons in the antennal lobe of the fly. Neuron 36: 463-474.

Noh J, Seal RP, Garver JA, Edwards RH, Kandler K. 2010. Glutamate co-release at GABA/glycinergic synapses is crucial for the refinement of an inhibitory map. Nat Neurosci 13: 232-238.

Okada R, Awasaki T, Ito K. 2009. Gamma-aminobutyric acid (GABA)-mediated neural connections in the Drosophila antennal lobe. J Comp Neurol 514: 74-91.

Pan Y, Zhou Y, Guo C, Gong H, Gong Z, Liu L. 2009. Differential roles of the fan-shaped body and the ellipsoid body in Drosophila visual pattern memory. Learn Mem 16: 289-295.

Pinsker H, Kupfermann I, Castellucci V, Kandel E. 1970. Habituation and dishabituation of the gill-withdrawal reflex in Aplysia. Science 167: $1740-1742$.

Pulver SR, Pashkovski SL, Hornstein NJ, Garrity PA, Griffith LC. 2009. Temporal dynamics of neuronal activation by Channelrhodopsin-2 and TRPA1 determine behavioral output in Drosophila larvae. J Neurophysiol 101: 3075-3088.

Quian Quiroga R, van Luijtelaar EL. 2002. Habituation and sensitization in rat auditory evoked potentials: A single-trial analysis with wavelet denoising. Int J Psychophysiol 43: 141-153.
Rankin CH, Abrams T, Barry RJ, Bhatnagar S, Clayton DF, Colombo J, Coppola G, Geyer MA, Glanzman DL, Marsland S, et al. 2009. Habituation revisited: An updated and revised description of the behavioral characteristics of habituation. Neurobiol Learn Mem 92: $135-138$.

Rees CT, Spatz HC. 1989. Habituation of the landing response of Drosophila wild-type and mutants defective in olfactory learning. J Neurogenet 5: $105-118$.

Rosenzweig M, Brennan KM, Tayler TD, Phelps PO, Patapoutian A, Garrity PA. 2005. The Drosophila ortholog of vertebrate TRPA1 regulates thermotaxis. Genes Dev 19: 419-424.

Scheiner R, Sokolowski MB, Erber J. 2004. Activity of cGMP-dependent protein kinase (PKG) affects sucrose responsiveness and habituation in Drosophila melanogaster. Learn Mem 11: 303-311.

Shiraiwa T, Carlson JR. 2007. Proboscis extension response (PER) assay in Drosophila. J Vis Exp 3: e193. doi: 10.3791/193.

Shirinyan D, Teshiba T, Taylor K, O'Neill P, Lee SC, Krasne FB. 2006. Rostral ganglia are required for induction but not expression of crayfish escape reflex habituation: Role of higher centers in reprogramming low-level circuits. J Neurophysiol 95: 2721-2724.

Singh RN. 1997. Neurobiology of the gustatory systems of Drosophila and some terrestrial insects. Microsc Res Tech 39: 547-563.

Stocker RF. 1994. The organization of the chemosensory system in Drosophila melanogaster: A review. Cell Tissue Res 275: 3-26.

Stopfer M, Carew TJ. 1996. Heterosynaptic facilitation of tail sensory neuron synaptic transmission during habituation in tail-induced tail and siphon withdrawal reflexes of Aplysia. J Neurosci 16: $4933-4948$.

Sudhakaran IP, Holohan EE, Osman S, Rodrigues V, VijayRaghavan K, Ramaswami M. 2012. Plasticity of recurrent inhibition in the Drosophila antennal lobe. J Neurosci 32: 7225-7231.

Suster ML, Bate M. 2002. Embryonic assembly of a central pattern generator without sensory input. Nature 416: 174-178.

Szabadits E, Cserep C, Szonyi A, Fukazawa Y, Shigemoto R, Watanabe M, Itohara S, Freund TF, Nyiri G. 2011. NMDA receptors in hippocampal GABAergic synapses and their role in nitric oxide signaling. J Neurosci 31: $5893-5904$.

Thompson RF, Spencer WA. 1966. Habituation: A model phenomenon for the study of neuronal substrates of behavior. Psychol Rev 73: 16-43.

Tully T, Quinn WG. 1985. Classical conditioning and retention in normal and mutant Drosophila melanogaster. J Comp Physiol A 157: 263-277.

Wang Z, Singhvi A, Kong P, Scott K. 2004. Taste representations in the Drosophila brain. Cell 117: 981-991.

Wilson DA. 2009. Olfaction as a model system for the neurobiology of mammalian short-term habituation. Neurobiol Learn Mem 92: 199-205.

Wilson DA, Sullivan RM, Leon M. 1985. Odor familiarity alters mitral cell response in the olfactory bulb of neonatal rats. Brain Res 354: 314-317.

Wolf FW, Eddison M, Lee S, Cho W, Heberlein U. 2007. GSK-3/Shaggy regulates olfactory habituation in Drosophila. Proc Natl Acad Sci 104: $4653-4657$.

Wu CL, Xia S, Fu TF, Wang H, Chen YH, Leong D, Chiang AS, Tully T. 2007. Specific requirement of NMDA receptors for long-term memory consolidation in Drosophila ellipsoid body. Nat Neurosci 10: $1578-1586$.

Xia S, Miyashita T, Fu TF, Lin WY, Wu CL, Pyzocha L, Lin IR, Saitoe M, Tully T, Chiang AS. 2005. NMDA receptors mediate olfactory learning and memory in Drosophila. Curr Biol 15: 603-615.

Yasuyama K, Salvaterra PM. 1999. Localization of choline acetyltransferase-expressing neurons in Drosophila nervous system. Microsc Res Tech 45: 65-79.

Yasuyama K, Meinertzhagen IA, Schurmann FW. 2003. Synaptic connections of cholinergic antennal lobe relay neurons innervating the lateral horn neuropile in the brain of Drosophila melanogaster. J Comp Neurol 466: 299-315.

Zars T, Fischer M, Schulz R, Heisenberg M. 2000a. Localization of a short-term memory in Drosophila. Science 288: 672-675.

Zars T, Wolf R, Davis R, Heisenberg M. 2000b. Tissue-specific expression of a type I adenylyl cyclase rescues the rutabaga mutant memory defect: In search of the engram. Learn Mem 7: 18-31.

Zordan MA, Massironi M, Ducato MG, Te Kronnie G, Costa R, Reggiani C, Chagneau C, Martin JR, Megighian A. 2005. Drosophila CAKI/CMG protein, a homolog of human CASK, is essential for regulation of neurotransmitter vesicle release. J Neurophysiol 94: 1074-1083.

Received April 10, 2012; accepted in revised form September 7, 2012. 


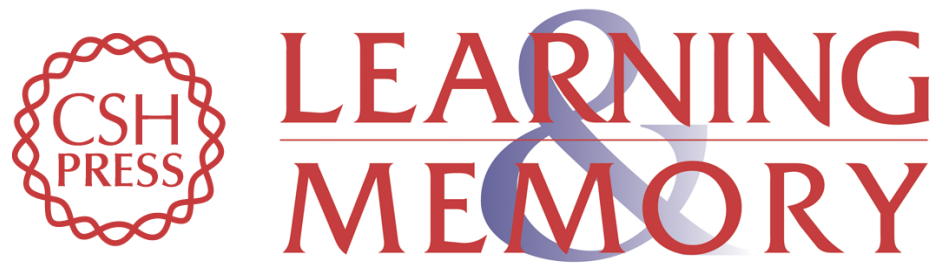

\section{Gustatory habituation in Drosophila relies on rutabaga (adenylate cyclase)-dependent plasticity of GABAergic inhibitory neurons}

Pushkar Paranjpe, Veronica Rodrigues, K. VijayRaghavan, et al.

Learn. Mem. 2012, 19:

Access the most recent version at doi:10.1101//m.026641.112 Supplemental
Material http://learnmem.cshlp.org/content/suppl/2012/11/20/19.12.627.DC1

References This article cites 67 articles, 26 of which can be accessed free at: http://learnmem.cshlp.org/content/19/12/627.full.html\#ref-list-1

License

Email Alerting Receive free email alerts when new articles cite this article - sign up in the box at the Service top right corner of the article or click here. 